\title{
A Conservative Crank-Nicolson Fourier Spectral Method for the Space Fractional Schrödinger Equation with Wave Operators
}

\author{
Lei Zhang $\mathbb{D}^{1}$, Rui Yang $\mathbb{D},{ }^{2}$ Li Zhang $\mathbb{D}^{3}$, and Lisha Wang $\mathbb{D}^{1}$ \\ ${ }^{1}$ Department of Mathematics, Qingdao University of Technology, Qingdao 266520, China \\ ${ }^{2}$ Foundational Courses Department, Hebei Vocational University of Scientific and Technical Engineering, Xingtai 054000, China \\ ${ }^{3}$ Jining Polytechnic, Jining 272000, China
}

Correspondence should be addressed to Rui Yang; yruimath@126.com

Received 10 April 2021; Accepted 27 May 2021; Published 9 June 2021

Academic Editor: Xinguang Zhang

Copyright (c) 2021 Lei Zhang et al. This is an open access article distributed under the Creative Commons Attribution License, which permits unrestricted use, distribution, and reproduction in any medium, provided the original work is properly cited.

In this paper, the Crank-Nicolson Fourier spectral method is proposed for solving the space fractional Schrödinger equation with wave operators. The equation is treated with the conserved Crank-Nicolson Fourier Galerkin method and the conserved CrankNicolson Fourier collocation method, respectively. In addition, the ability of the constructed numerical method to maintain the conservation of mass and energy is studied in detail. Meanwhile, the convergence with spectral accuracy in space and secondorder accuracy in time is verified for both Galerkin and collocation approximations. Finally, the numerical experiments verify the properties of the conservative difference scheme and demonstrate the correctness of theoretical results.

\section{Introduction}

The Schrödinger equation is one of the most basic equations in quantum mechanics, which was proposed by Austrian physicist Schrödinger in 1926. The equation can correctly describe the quantum behaviors of wave function, which has made great contributions to the study of quantum mechanics. Since then, the Schrödinger system has attracted a large number of mathematicians and physicists to explore the characteristics of its solution and physical applications. The study of conservative methods for the Schrödinger equation is one of the most popular research fields.

Over the past decades, most of the researches on the conservative method of the Schrödinger equation focus on the integerorder Schrödinger equation (e.g., see Refs. [1-7]). As models of science and engineering are needed to be more realistic, the fractional-order Schrödinger equation becomes one of the most important models in the fields of Bose-Einstein condensation, plasma, nonlinear optics, fluid dynamics [8, 9], etc. However, few studies have been investigated on conservative methods for the fractional Schrödinger equation. Besides that, most of the existing fractional-order conservative methods are finite element and finite difference methods $[10,11]$.
From the viewpoint of mathematics, the solution of the Schrödinger system has important geometric structures such as energy conservation and multisymplectic structure. Therefore, these properties should be maintained as much as possible in the construction of numerical methods. In this paper, we consider the following nonlinear fractional Schrödinger equation:

$$
\begin{aligned}
& \phi_{t t}(y, t)+(-\Delta)^{\alpha / 2} \phi(y, t)+i \kappa \phi_{t}(y, t) \\
& \quad+\beta|\phi(y, t)|^{2} \phi(y, t)=0, \quad y \in(a, b), 0<t \leq T,
\end{aligned}
$$

subject to the boundary condition

$$
\phi(a, t)=\phi(b, t), \quad 0<t \leq T,
$$

and the initial conditions

$$
\phi(y, 0)=\phi_{0}(y), \phi_{t}(y, 0)=\phi_{1}(y), \quad y \in(a, b),
$$

where $\beta$ and $\kappa$ are positive real constants, $1<\alpha \leq 2$, and $i^{2}$ $=-1 . \phi_{0}(y)$ and $\phi_{1}(y)$ are given real functions. The fractional 
Laplacian operator $(-\Delta)^{\alpha / 2}$ can be defined as a pseudodifferential operator with the symbol $-|\xi|^{\alpha}$ :

$$
-(-\Delta)^{\alpha / 2} u(x, t):=-\mathscr{F}^{-1}\left(|\xi|^{\alpha} \widehat{\mathcal{u}}(\xi, t)\right),
$$

where $\mathscr{F}$ is the Fourier transform and $\widehat{u}$ is the Fourier transform of $u$.

The spectral method is a generalization of a standard separation variable method, for which Chebyshev polynomials and Legendre polynomials are generally used as the basic functions of approximate expansions. And the Fourier series is convenient to deal with the periodic boundary conditions. Bridges and Reich [12] first put forward the Hamiltonian system using the Fourier spectrum discrete method in 2001. Based on their theoretical ideas, Chen and Qin [13] in the same year proposed the Fourier pseudo-spectral method for the Hamiltonian partial differential equation and used it to integrate the nonlinear Schrödinger equation with periodic boundary conditions. For more comprehensive work on the different conservative Fourier pseudo-spectral methods, refer to $[2,14-16]$ and their references.

Since the equation is calculated on a finite interval $[a, b]$, it is converted into periodic boundary conditions in this paper and studied on $\Omega=[0,2 \pi]$ and $I=[0, T]$ below. Let

$$
x=\frac{(2 y-a-b) \pi}{b-a}+\pi, \quad x \in[0,2 \pi] .
$$

Denote $u(x, t)=\phi(y, t), u(0, t)=u(2 \pi, t), u_{0}(x)=\phi_{0}(y)$, and $u_{1}(x)=\phi_{1}(y)$. Thus, (1)-(3) become

$$
\begin{gathered}
u_{t t}(x, t)+M^{\alpha}(-\Delta)^{\alpha / 2} u(x, t)+i \kappa u_{t}(x, t) \\
+\beta|u(x, t)|^{2} u(x, t)=0, \quad x \in(0,2 \pi), 0<t \leq T, \\
u(0, t)=u(2 \pi, t), \\
u(x, 0)=u_{0}(x), u_{t}(x, 0)=u_{1}(x), \quad x \in(0,2 \pi),
\end{gathered}
$$

where $M=2 \pi /(b-a)$.

In fact, the nonlinear fractional Schrödinger equation ((6)-(8)) has two conserved quantities:

$$
Q(t)=Q(0), E(t)=E(0), \quad 0 \leq t \leq T,
$$

where

$$
\begin{gathered}
Q(t)=\frac{\kappa}{2}\|u(\cdot, t)\|_{L^{2}}^{2}+\operatorname{Im}\left(u_{t}, u\right), \\
E(t)=\left\|u_{t}(\cdot, t)\right\|_{L^{2}}^{2}+M^{\alpha}\left\|(-\Delta)^{\alpha / 4} u(\cdot, t)\right\|_{L^{2}}^{2}+\frac{\beta}{2}\|u(\cdot, t)\|_{L^{4}}^{4},
\end{gathered}
$$

with

$$
\|u(\cdot, t)\|_{L^{p}}^{p}=\int_{\Omega}|u(x, t)|^{p} d x, \quad p=2,4 .
$$

The outline of the remainder of this paper is as follows. In Section 2, a conserved Crank-Nicolson Fourier Galerkin method and a conserved Crank-Nicolson Fourier collocation method are constructed to discrete time variables and spatial variables. Energy-preserving and mass-preserving properties of the new method are investigated, and the error estimate is derived in Section 3. In Section 4, numerical experiments are presented to illustrate the theoretical results. Finally, the conclusions are given in Section 5.

\section{Crank-Nicolson Fourier Spectral Method and Conservation Laws}

Let $C_{\text {per }}^{\infty}(\Omega)$ be the set of all complex-valued and $2 \pi$-periodic $C^{\infty}$-functions on $\Omega$. Denote $(\cdot, \cdot)$ as the inner product on the space $L_{\text {per }}^{2}(\Omega)$ with the $L^{2}$ norm $\|\cdot\|_{L_{\text {per }}^{2}(\Omega)}($ abbreviated as $\|\cdot\|)$ :

$$
\begin{aligned}
(u(x), v(x)) & =\int_{\Omega} u(x) \bar{v}(x) d x, \\
\|u(x)\|_{L_{\text {per }}^{2}(\Omega)} & =\sqrt{(u(x), u(x))} .
\end{aligned}
$$

For $\mu$ as a nonnegative real number, let $H_{\text {per }}^{\mu}(\Omega)$ be the closure of $C_{\text {per }}^{\infty}(\Omega)$. Note that $H_{\text {per }}^{0}(\Omega)=L_{\text {per }}^{2}(\Omega)$. For any function $u(x) \in L_{\text {per }}^{2}(\Omega)$, the following equations [17] can be developed easily:

$$
u(x)=\sum_{\omega \in \mathbb{Z}} \widehat{u}_{\omega} e^{i \omega x},
$$

where the Fourier coefficients are arranged as

$$
\widehat{u}_{\omega}=\left(u, e^{i \omega x}\right)=\frac{1}{2 \pi} \int_{\Omega} u e^{-i \omega x} d x
$$

For the Fourier transform of fractional Laplacian $(-\Delta)^{\alpha / 2}$, we have

$$
\mathscr{F}\left\{-(-\Delta)^{\alpha / 2} u(x, t)\right\}(\omega)=-|\omega|^{\alpha} \mathscr{F}\{u(x, t)\}(\omega) .
$$

In order to discretize the equation in the temporal direction, the time step is defined by $\tau=T / N_{t}$. Denote difference operator

$$
\begin{aligned}
\delta_{t}^{2} u^{n} & =\frac{u^{n+1}-2 u^{n}+u^{n-1}}{\tau^{2}}, \delta_{t} u^{n}=\frac{u^{n+1}-u^{n}}{\tau}, \delta_{\hat{t}} u^{n} \\
& =\frac{u^{n+1}-u^{n-1}}{2 \tau}, \tilde{u}^{n}=\frac{u^{n-1}+u^{n+1}}{2},
\end{aligned}
$$

where $n$ is a positive integer $\left(0 \leq n \leq N_{t}\right)$. Therefore, the Crank-Nicolson method was used to discretize equation (6) in the time axis. 


$$
\begin{aligned}
i \kappa \delta_{\hat{t}} u^{n}= & -\delta_{t}^{2} u^{n}-M^{\alpha}(-\Delta)^{\alpha / 2} \tilde{u}^{n} \\
& -\frac{\beta}{2}\left(\left|u^{n-1}\right|^{2}+\left|u^{n+1}\right|^{2}\right) \tilde{u}^{n}+R^{n},
\end{aligned}
$$

where $R^{n}=O\left(\tau^{2}\right)$.

2.1. Crank-Nicolson Fourier Galerkin Method. For positive even number $N$, the basis function space can be constructed as

$$
S_{N}=\operatorname{span}\left\{\mathrm{e}^{i \omega x} \mid-\frac{N}{2} \leq \omega \leq \frac{N}{2}-1\right\},
$$

where the norm and seminorm of $H_{\text {per }}^{\alpha}(\Omega)$ are characterized by

$$
\begin{aligned}
& \|u\|_{\alpha} \triangleq\left(\sum_{\omega=-N / 2}^{(N / 2)-1}\left(1+|\omega|^{2 \alpha}\right)\left|\widehat{u}_{\omega}\right|^{2}\right)^{1 / 2} \\
& |u|_{\alpha} \triangleq\left(\sum_{\omega=-N / 2}^{(N / 2)-1}|\omega|^{2 \alpha}\left|\widehat{u}_{\omega}\right|^{2}\right)^{1 / 2} .
\end{aligned}
$$

Let

$$
u_{N}(t)=\sum_{\omega=-N / 2}^{(N / 2)-1} \widehat{u}_{\omega}(t) \mathrm{e}^{i \omega x}
$$

The orthogonal operators $P_{N}: L_{\text {per }}^{2}(\Omega) \rightarrow S_{N}$ are defined as follows:

$$
\left(P_{N} u-u, v\right)=0, \quad \forall v \in S_{N}
$$

Lemma $1[18,19]$. Suppose that $u \in H_{p e r}^{s}(\Omega)$ for all $0 \leq \mu \leq s$; it holds that

$$
\left\|u-P_{N} u\right\|_{\mu} \leq C N^{\mu-s}\|u\|_{s} .
$$

Denote

$$
u_{N}^{n}=\sum_{\omega=-N / 2}^{(N / 2)-1} \widehat{u}_{\omega}^{n} e^{i \omega x}, \quad n=0,1, \cdots, N_{t}
$$

The time variables of equation (6) are discretized by the Crank-Nicolson method. And the discrete Fourier Galerkin approximation for equation (6) has a modified scheme as follows:

$$
\begin{aligned}
& \left(\tau \kappa u_{N}^{n+1}-\tau^{2} M^{\alpha} i(-\Delta)^{\alpha / 2} u_{N}^{n+1}-2 i u_{N}^{n+1}, v\right) \\
& \quad=\left(\tau \kappa u_{N}^{n-1}+\tau^{2} M^{\alpha} i(-\Delta)^{\alpha / 2} u_{N}^{n-1}+2 i u_{N}^{n-1}, v\right) \\
& \quad-\left(4 i u_{N}^{n}, v\right)+\frac{\beta \tau^{2} i}{2}\left(\left(\left|u_{N}^{n+1}\right|^{2} u_{N}^{n+1}+\left|u_{N}^{n-1}\right|^{2} u_{N}^{n-1}\right.\right. \\
& \left.\left.\quad+\left|u_{N}^{n+1}\right|^{2} u_{N}^{n-1}+\left|u_{N}^{n-1}\right|^{2} u_{N}^{n+1}\right), v\right)
\end{aligned}
$$

$$
u_{N}^{0}=P_{N} u_{0}, \delta_{\bar{t}} u_{N}^{0}=P_{N} u_{1},
$$

where $u_{N}^{n+1} \in S_{N}, \forall v \in S_{N}$.

2.2. Crank-Nicolson Fourier Collocation Method. For positive even number $N$, consider the points $x_{j}=2 \pi j / N, j=0,1, \cdots$, $N-1$, as collocation nodes. The discrete Fourier coefficients [18] of a function $u$ on $[0,2 \pi]$ with respect to the collocation points are the following form:

$$
\widehat{u}_{\omega}=\frac{1}{N} \sum_{j=0}^{N-1} u\left(x_{j}\right) e^{-i \omega x_{j}}, \quad \omega=-\frac{N}{2}, \cdots, \frac{N}{2}-1 .
$$

Using the inversion formula, we have

$$
u\left(x_{j}\right)=\sum_{\omega=-N / 2}^{(N / 2)-1} \widehat{u}_{\omega} \mathrm{e}^{i \omega x_{j}}, \quad j=0,1, \cdots, N-1 .
$$

Define the interpolation operator $I_{N}[18]$ at the collocation points:

$$
I_{N} u\left(x_{j}\right)=u\left(x_{j}\right), \quad j=0,1, \cdots, N-1
$$

According to (27),

$$
\left(I_{N} u\right)(x)=\sum_{\omega=-N / 2}^{(N / 2)-1} \widehat{u}_{\omega} \mathrm{e}^{i \omega x}
$$

Lemma $2[18,19]$. For any $u \in H_{\text {per }}^{s}(\Omega), s \geq 1$, the estimate

$$
\left\|u-I_{N} u\right\| \leq C N^{-s}\|u\|_{s}
$$

in the sense of the Sobolev norm.

Combining Lemma 2 and the triangle inequality, Corollary 3 is drawn.

Corollary 3. For any $u \in H_{\text {per }}^{s}(\Omega), s \geq 1$, there exists a constant $C$ independent of $u$ and $N$, such that

$$
\left\|I_{N} u\right\| \leq C N^{-s}\|u\|_{s}+\|u\|
$$

Using the Fourier collocation method to discrete the spatial variables of the equation, we get the fully discrete scheme for equations (6)-(8) as the following forms:

$$
\begin{aligned}
\tau & \kappa u_{N}^{n+1}\left(x_{j}\right)-\tau^{2} M^{\alpha} i(-\Delta)^{\alpha / 2} u_{N}^{n+1}\left(x_{j}\right)-2 i u_{N}^{n+1}\left(x_{j}\right) \\
= & \tau \kappa u_{N}^{n-1}\left(x_{j}\right)+\tau^{2} M^{\alpha} i(-\Delta)^{\alpha / 2} u_{N}^{n-1}\left(x_{j}\right) \\
& +2 i u_{N}^{n-1}\left(x_{j}\right)-4 i u_{N}^{n}\left(x_{j}\right)+\frac{\beta \tau^{2} i}{2} I_{N} \\
& \cdot\left(\left|u_{N}^{n+1}\left(x_{j}\right)\right|^{2} u_{N}^{n+1}\left(x_{j}\right)+\left|u_{N}^{n-1}\left(x_{j}\right)\right|^{2} u_{N}^{n-1}\left(x_{j}\right)\right) \\
& +\frac{\beta \tau^{2} i}{2} I_{N}\left(\left|u_{N}^{n+1}\left(x_{j}\right)\right|^{2} u_{N}^{n-1}\left(x_{j}\right)+\left|u_{N}^{n-1}\left(x_{j}\right)\right|^{2} u_{N}^{n+1}\left(x_{j}\right)\right),
\end{aligned}
$$




$$
u_{N}^{0}\left(x_{j}\right)=u_{0}\left(x_{j}\right), \delta_{\hat{t}} u_{N}^{0}\left(x_{j}\right)=u_{1}\left(x_{j}\right) .
$$

Applying the Fourier transformation to (24), we get the following form:

$$
\begin{aligned}
\left(\tau \kappa-\tau^{2} M^{\alpha} i|\omega|^{\alpha}-2 i\right) \widehat{u}_{N \omega}^{n+1}= & \left(\tau \kappa+\tau^{2} M^{\alpha} i|\omega|^{\alpha}+2 i\right) \widehat{u}_{N \omega}^{n-1} \\
& -4 i \widehat{u}_{N \omega}^{n}+\frac{\beta \tau^{2} i}{2} \widehat{F}_{N \omega}^{n}, \\
\left(\widehat{u_{N}^{0}}\right)_{\omega}= & \left(\widehat{u_{0}}\right)_{\omega},\left(\widehat{\delta_{t} \hat{u}_{N}^{0}}\right)_{\omega}=\left(\widehat{u_{1}}\right)_{\omega},
\end{aligned}
$$

where $F_{N}^{n}=\left(\left|u_{N}^{n+1}\right|^{2} u_{N}^{n+1}+\left|u_{N}^{n-1}\right|^{2} u_{N}^{n-1}+\left|u_{N}^{n+1}\right|^{2} u_{N}^{n-1}+\left|u_{N}^{n-1}\right|^{2}\right.$ $\left.u_{N}^{n+1}\right), \omega=-N / 2, \cdots N / 2-1$.

\subsection{Theory Analysis of Conservation}

Theorem 4. The Crank-Nicolson Fourier Galerkin method (24) of solving equations (6)-(8) preserves the discrete mass and discrete energy:

$$
\begin{array}{ll}
Q^{n}=Q^{0}, & 0 \leq n \leq N_{t}, \\
E^{n}=E^{0}, & 0 \leq n \leq N_{t},
\end{array}
$$

where

$$
\begin{aligned}
Q^{n}= & \operatorname{Im}\left(\delta_{t} u_{N}^{n}, u_{N}^{n}\right)+\frac{\kappa}{4}\left(\left\|u_{N}^{n+1}\right\|^{2}+\left\|u_{N}^{n}\right\|^{2},\right. \\
E^{n}= & \left\|\delta_{t} u_{N}^{n}\right\|^{2}+\frac{M^{\alpha}}{2}\left(\left\|(-\Delta)^{\alpha / 4} u_{N}^{n+1}\right\|^{2}+\left\|(-\Delta)^{\alpha / 4} u_{N}^{n}\right\|^{2}\right) \\
& +\frac{\beta}{4}\left(\left\|u_{N}^{n+1}\right\|_{L^{4}(\Omega)}^{4}+\left\|u_{N}^{n}\right\|_{L^{4}(\Omega)}^{4}\right) .
\end{aligned}
$$

Proof. We derive the full discrete Fourier Galerkin method:

$$
\begin{aligned}
\left(i \kappa \delta_{\hat{t}} u_{N}^{n}, v\right)= & -\left(\delta_{t}^{2} u_{N}^{n}, v\right)-M^{\alpha}(-\Delta)^{\alpha / 2}\left(\tilde{u}_{N}^{n}, v\right) \\
& -\frac{\beta}{2}\left(\left(\left|u_{N}^{n-1}\right|^{2}+\left|u_{N}^{n+1}\right|^{2}\right) \tilde{u}_{N}^{n}, v\right) .
\end{aligned}
$$

Let $v=\tilde{u}_{N}^{n}$ in equation (37); it holds that

$$
\begin{aligned}
\left(i \kappa \delta_{\hat{t}} u_{N}^{n}, \tilde{u}_{N}^{n}\right)= & -\left(\delta_{t}{ }^{2} u_{N}^{n}, \tilde{u}_{N}^{n}\right)-M^{\alpha}\left((-\Delta)^{\alpha / 2} \tilde{u}_{N}^{n}, \tilde{u}_{N}^{n}\right) \\
& -\frac{\beta}{2}\left(\left(\left|u_{N}^{n-1}\right|^{2}+\left|u_{N}^{n+1}\right|^{2}\right) \tilde{u}_{N}^{n}, \tilde{u}_{N}^{n}\right) .
\end{aligned}
$$

Taking the imaginary part of equation (38), due to

$$
\begin{aligned}
& \operatorname{Im}\left(i \kappa \delta_{\hat{t}} u_{N}^{n}, \tilde{u}_{N}^{n}\right)= \kappa \operatorname{Re}\left(\delta_{\hat{t}} u_{N}^{n}, \tilde{u}_{N}^{n}\right) \\
&= \kappa \operatorname{Re}\left(\frac{u_{N}^{n+1}-u_{N}^{n-1}}{2 \tau}, \frac{u_{N}^{n-1}+u_{N}^{n+1}}{2}\right) \\
&= \frac{\kappa}{4 \tau}\left(\left\|u_{N}^{n+1}\right\|^{2}-\|\left. u_{N}^{n-1}\right|^{2}\right), \\
& \operatorname{Im}\left(\delta_{t}^{2} u_{N}^{n}, \tilde{u}_{N}^{n}\right)= \frac{1}{2 \tau}\left[\operatorname{Im}\left(\delta_{t} u_{N}^{n}, u_{N}^{n-1}+u_{N}^{n+1}\right)\right. \\
&\left.-\operatorname{Im}\left(\delta_{t} u_{N}^{n-1}, u_{N}^{n-1}+u_{N}^{n+1}\right)\right] \\
&= \frac{1}{2 \tau}\left[2 \operatorname{Im}\left(\delta_{t} u_{N}^{n}, u_{N}^{n}\right)+\operatorname{Im}\left(\delta_{t} u_{N}^{n}, u_{N}^{n+1}-u_{N}^{n}\right)\right. \\
&-\operatorname{Im}\left(\delta_{t} u_{N}^{n}, u_{N}^{n}-u_{N}^{n-1}\right)-\operatorname{Im}\left(\delta_{t} u_{N}^{n-1}, u_{N}^{n+1}-u_{N}^{n}\right) \\
&-\operatorname{Im}\left(\delta_{t} u_{N}^{n-1}, u_{N}^{n}-u_{N}^{n-1}\right)-2 \operatorname{Im}\left(\delta_{t} u_{N}^{n-1}, u_{N}^{n-1}\right) \\
&= \frac{1}{\tau}\left[\operatorname{Im}\left(\delta_{t} u_{N}^{n}, u_{N}^{n}\right)-\operatorname{Im}\left(\delta_{t} u_{N}^{n-1}, u_{N}^{n-1}\right)\right], \\
& \operatorname{Im}\left((-\Delta)^{\alpha / 2} \tilde{u}_{N}^{n}, \tilde{u}_{N}^{n}\right)=0, \\
& \operatorname{Im}\left(\left(\left|u_{N}^{n-1}\right|^{2}+\left|u_{N}^{n+1}\right|^{2}\right) \tilde{u}_{N}^{n}, \tilde{u}_{N}^{n}\right)=0 .
\end{aligned}
$$

Therefore,

$$
\frac{\operatorname{Im}\left(\delta_{t} u_{N}^{n}, u_{N}^{n}\right)-\operatorname{Im}\left(\delta_{t} u_{N}^{n-1}, u_{N}^{n-1}\right)}{\tau}
$$

thus,

$$
\begin{aligned}
& \operatorname{Im}\left(\delta_{t} u_{N}^{n}, u_{N}^{n}\right)+\frac{\kappa}{4}\left(\left\|u_{N}^{n+1}\right\|^{2}+\left\|u_{N}^{n}\right\|^{2}\right) \\
& =\operatorname{Im}\left(\delta_{t} u_{N}^{n-1}, u_{N}^{n-1}\right)+\frac{\kappa}{4}\left(\left\|u_{N}^{n}\right\|^{2}+\left\|u_{N}^{n-1}\right\|^{2}\right) .
\end{aligned}
$$

The above equality indicates that the method (24) maintains the conservation of the discrete mass. The following items consider the conservation of the discrete energy.

Let $v=\delta_{\widehat{t}} u_{N}^{n}$; according to equation (37), we also get

$$
\begin{aligned}
\left(i \kappa \delta_{\hat{t}} u_{N}^{n}, \delta_{\widehat{t}} u_{N}^{n}\right)= & -\left(\delta_{t}^{2} u_{N}^{n}, \delta_{\widehat{t}} u_{N}^{n}\right)-M^{\alpha}\left((-\Delta)^{\alpha / 2} \tilde{u}_{N}^{n}, \delta_{\widehat{t}} u_{N}^{n}\right) \\
& -\frac{\beta}{2}\left(\left(\left|u_{N}^{n-1}\right|^{2}+\left|u_{N}^{n+1}\right|^{2}\right) \tilde{u}_{N}^{n}, \delta_{\widehat{t}} u_{N}^{n}\right) .
\end{aligned}
$$

Taking the real part of (42), due to

$$
\operatorname{Re}\left(i \kappa \delta_{\widehat{t}} u_{N}^{n}, \delta_{\widehat{t}} u_{N}^{n}\right)=0,
$$

$$
\begin{aligned}
\operatorname{Re}\left(\delta_{t}{ }^{2} u_{N}^{n}, \delta_{\vec{t}} u_{N}^{n}\right) & =\frac{1}{2 \tau} \operatorname{Re}\left(\delta_{t} u_{N}^{n}-\delta_{t} u_{N}^{n-1}, \delta_{t} u_{N}^{n}+\delta_{t} u_{N}^{n-1}\right) \\
& =\frac{\left\|\delta_{t} u_{N}^{n}\right\|^{2}-\left\|\delta_{t} u_{N}^{n-1}\right\|^{2}}{2 \tau},
\end{aligned}
$$




$$
\begin{aligned}
& \operatorname{Re}\left((-\Delta)^{\alpha / 2} \tilde{u}_{N}^{n}, \delta_{\hat{t}} u_{N}^{n}\right) \\
&= \operatorname{Re}\left((-\Delta)^{\alpha / 2} \frac{u_{N}^{n-1}+u_{N}^{n+1}}{2}, \frac{u_{N}^{n+1}-u_{N}^{n-1}}{2 \tau}\right) \\
&= \frac{1}{4 \tau}\left(\left((-\Delta)^{\alpha / 4} u_{N}^{n+1},(-\Delta)^{\alpha / 4} u_{N}^{n+1}\right)\right. \\
&\left.-\left((-\Delta)^{\alpha / 4} u_{N}^{n-1},(-\Delta)^{\alpha / 4} u_{N}^{n-1}\right)\right) \\
&= \frac{\left\|(-\Delta)^{\alpha / 4} u_{N}^{n+1}\right\|^{2}-\left\|(-\Delta)^{\alpha / 4} u_{N}^{n-1}\right\|^{2}}{4 \tau}, \\
& \operatorname{Re}\left(\left(\left|u_{N}^{n-1}\right|^{2}+\left|u_{N}^{n+1}\right|^{2}\right) \tilde{u}_{N}^{n}, \delta_{\hat{t}} u_{N}^{n}\right) \\
&=\operatorname{Re}\left(\left(\left|u_{N}^{n-1}\right|^{2}+\left|u_{N}^{n+1}\right|^{2}\right) \frac{u_{N}^{n-1}+u_{N}^{n+1}}{2}, \frac{u_{N}^{n+1}-u_{N}^{n-1}}{2 \tau}\right) \\
&=\frac{1}{4 \tau}\left(\left\|u_{N}^{n+1}\right\|_{L^{4}(\Omega)}^{4}-\left\|u_{N}^{n-1}\right\|_{L^{4}(\Omega)}^{4}\right),
\end{aligned}
$$

therefore, using (43)-(46), we obtain

$$
\begin{aligned}
& \frac{\left\|\delta_{t} u_{N}^{n}\right\|^{2}-\left\|\delta_{t} u_{N}^{n-1}\right\|^{2}}{2 \tau}+\frac{M^{\alpha}}{4 \tau}\left(\left\|(-\Delta)^{\alpha / 4} u_{N}^{n+1}\right\|^{2}\right. \\
& \left.-\left\|(-\Delta)^{\alpha / 4} u_{N}^{n-1}\right\|^{2}\right)+\frac{\beta}{8 \tau}\left(\left\|u_{N}^{n+1}\right\|_{L^{4}(\Omega)}^{4}-\left\|u_{N}^{n-1}\right\|_{L^{4}(\Omega)}^{4}\right)=0,
\end{aligned}
$$

thus,

$$
\begin{aligned}
\left\|\delta_{t} u_{N}^{n}\right\|^{2}+\frac{M^{\alpha}}{2}\left(\left\|(-\Delta)^{\alpha / 4} u_{N}^{n+1}\right\|^{2}+\left\|(-\Delta)^{\alpha / 4} u_{N}^{n}\right\|^{2}\right) \\
+\frac{\beta}{4}\left(\left\|u_{N}^{n+1}\right\|_{L^{4}(\Omega)}^{4}+\left\|u_{N}^{n}\right\|_{L^{4}(\Omega)}^{4}\right) \\
=\left\|\delta_{t} u_{N}^{n-1}\right\|^{2}+\frac{M^{\alpha}}{2}\left(\left\|(-\Delta)^{\alpha / 4} u_{N}^{n}\right\|^{2}+\left\|(-\Delta)^{\alpha / 4} u_{N}^{n-1}\right\|^{2}\right) \\
+\frac{\beta}{4}\left(\left\|u_{N}^{n}\right\|_{L^{4}(\Omega)}^{4}+\left\|u_{N}^{n-1}\right\|_{L^{4}(\Omega)}^{4}\right) .
\end{aligned}
$$

Based on the above analysis, the method (24) also maintains the conservation of the discrete energy.

\section{Theory Analysis of Convergence}

In order to simplify the notation, we always assume that $C$ is a positive constant in this article, which might be different in every formula.

Lemma 5 [20]. For any discrete function $u_{N}^{n}$, it holds that

$$
\left\|u_{N}^{n+1}\right\|^{2}-\left\|u_{N}^{n}\right\|^{2} \leq \tau\left(\left\|\delta_{t} u_{N}^{n}\right\|^{2}+\frac{1}{2}\left(\left\|u_{N}^{n+1}\right\|^{2}+\left\|u_{N}^{n}\right\|^{2}\right)\right) .
$$

Lemma 6. For $u_{N} \in H_{\text {per }}^{\mu}(\Omega)$, there exists a positive constant $C$, such that

$$
\left\|u_{N}^{n}\right\| \leq C,\left\|(-\Delta)^{\alpha / 4} u_{N}^{n}\right\| \leq C,\left\|\delta_{t} u_{N}^{n}\right\| \leq C, \quad n=0,1, \cdots, N_{t} .
$$

Proof. Using Theorem 4, it yields

$$
\begin{gathered}
\left\|\delta_{t} u_{N}^{n}\right\|^{2}+\frac{M^{\alpha}}{2}\left(\left\|(-\Delta)^{\alpha / 4} u_{N}^{n+1}\right\|^{2}+\left\|(-\Delta)^{\alpha / 4} u_{N}^{n}\right\|^{2}\right) \\
+\frac{\beta}{4}\left(\left\|u_{N}^{n+1}\right\|_{L^{4}(\Omega)}^{4}+\left\|u_{N}^{n}\right\|_{L^{4}(\Omega)}^{4}\right)=E^{n}=E^{0},
\end{gathered}
$$

thus,

$$
\begin{gathered}
\left\|\delta_{t} u_{N}^{n}\right\|^{2}+\frac{M^{\alpha}}{2}\left(\left\|(-\Delta)^{\alpha / 4} u_{N}^{n+1}\right\|^{2}+\left\|(-\Delta)^{\alpha / 4} u_{N}^{n}\right\|^{2}\right) \\
=E^{0}-\frac{\beta}{4}\left(\left\|u_{N}^{n+1}\right\|_{L^{4}(\Omega)}^{4}+\left\|u_{N}^{n}\right\|_{L^{4}(\Omega)}^{4}\right) .
\end{gathered}
$$

Because of $\beta>0$, it satisfies

$\left\|\delta_{t} u_{N}^{n}\right\|^{2}+\frac{M^{\alpha}}{2}\left(\left\|(-\Delta)^{\alpha / 4} u_{N}^{n+1}\right\|^{2}+\left\|(-\Delta)^{\alpha / 4} u_{N}^{n}\right\|^{2}\right) \leq C$.

Sum the inequalities of Lemma 5 from 0 to $n$ yields

$$
\begin{aligned}
\left(1-\frac{\tau}{2}\right)\left\|u_{N}^{n+1}\right\|^{2} \leq & \left(1+\frac{\tau}{2}\right)\left\|u_{N}^{0}\right\|^{2}+\tau\left\|\delta_{t} u_{N}^{0}\right\|^{2} \\
& +\tau \sum_{k=1}^{n}\left(\left\|\delta_{t} u_{N}^{k}\right\|^{2}+\left\|u_{N}^{k}\right\|^{2}\right) .
\end{aligned}
$$

Adding (53) and (54), we can obtain the following items:

$$
\begin{aligned}
& \left(1-\frac{\tau}{2}\right)\left\|u_{N}^{n+1}\right\|^{2}+\left\|\delta_{t} u_{N}^{n}\right\|^{2} \\
& \quad+\frac{M^{\alpha}}{2}\left(\left\|(-\Delta)^{\alpha / 4} u_{N}^{n+1}\right\|^{2}+\left\|(-\Delta)^{\alpha / 4} u_{N}^{n}\right\|^{2}\right) \\
& \leq C+\tau \sum_{k=1}^{n}\left(\left\|\delta_{t} u_{N}^{k}\right\|^{2}+\left\|u_{N}^{k}\right\|^{2}\right) .
\end{aligned}
$$

For $\tau$ is sufficiently small $(\tau<1)$, this implies

$$
\begin{aligned}
\frac{1}{2}\left\|u_{N}^{n+1}\right\|^{2}+\left\|\delta_{t} u_{N}^{n}\right\|^{2} & \\
& +\frac{M^{\alpha}}{2}\left(\left\|(-\Delta)^{\alpha / 4} u_{N}^{n+1}\right\|^{2}+\left\|(-\Delta)^{\alpha / 4} u_{N}^{n}\right\|^{2}\right) \\
\leq & C+2 \tau \sum_{k=1}^{n}\left(\frac{1}{2}\left\|u_{N}^{k}\right\|^{2}+\left\|\delta_{t} u_{N}^{k-1}\right\|^{2}\right. \\
& \left.+\frac{M^{\alpha}}{2}\left(\left\|(-\Delta)^{\alpha / 4} u_{N}^{k}\right\|^{2}+\left\|(-\Delta)^{\alpha / 4} u_{N}^{k-1}\right\|^{2}\right)\right) .
\end{aligned}
$$


According to the discrete Gronwall's inequality, there is

$$
\begin{gathered}
\frac{1}{2}\left\|u_{N}^{n+1}\right\|^{2}+\left\|\delta_{t} u_{N}^{n}\right\|^{2}+\frac{M^{\alpha}}{2}\left(\left\|(-\Delta)^{\alpha / 4} u_{N}^{n+1}\right\|^{2}\right. \\
\left.+\left\|(-\Delta)^{\alpha / 4} u_{N}^{n}\right\|^{2}\right) \leq C .
\end{gathered}
$$

Therefore,

$$
\left\|u_{N}^{n}\right\| \leq C,\left\|(-\Delta)^{\alpha / 4} u_{N}^{n}\right\|^{2} \leq C,\left\|\delta_{t} u_{N}^{n}\right\| \leq C
$$

Theorem 7. If $s \geq 1$, assume that $u \in C^{2}\left(I ; H_{\text {per }}^{\alpha}(\Omega) \cap H^{s}(\Omega)\right)$ is the exact solution of (6)-(8), and $u_{N}^{n}$ is the numerical solution of (24). It possesses the following conclusion:

$$
\left\|u_{N}^{n}-u\left(x, t_{n}\right)\right\| \leq C\left(\tau^{2}+N^{-s}\|u\|_{s}\right)
$$

Proof. Let $u^{*}=P_{N} u, e=u-u_{N}, \xi=u-u^{*}$, and $\eta=u^{*}-u_{N}$; then, $e^{n}=\xi^{n}+\eta^{n}$. From triangle inequality and Lemma 1, it yields

$$
\left\|e^{n}\right\| \leq\left\|\xi^{n}\right\|+\left\|\eta^{n}\right\| \leq C N^{-s}\|u\|_{s}+\left\|\eta^{n}\right\|
$$

According to the orthogonality of the projection operator $P_{N}$, we get

$$
\begin{aligned}
i\left(\kappa \delta_{\hat{t}} u^{* n}, v\right)= & -\left(\delta_{t}{ }^{2} u^{n}, v\right)-M^{\alpha}\left((-\Delta)^{\alpha / 2} \tilde{u}^{n}, v\right) \\
& -\frac{\beta}{2}\left(\left(\left|u^{n-1}\right|^{2}+\left|u^{n+1}\right|^{2}\right) \tilde{u}^{n}, v\right)+\left(R^{n}, v\right) .
\end{aligned}
$$

The authors derive the full discrete Fourier Galerkin method:

$$
\begin{aligned}
\left(i \kappa \delta_{\hat{t}} u_{N}^{n}, v\right)= & -\left(\delta_{t}^{2} u_{N}^{n}, v\right)-M^{\alpha}\left((-\Delta)^{\alpha / 2} \tilde{u}_{N}^{n}, v\right) \\
& -\frac{\beta}{2}\left(\left(\left|u_{N}^{n-1}\right|^{2}+\left|u_{N}^{n+1}\right|^{2}\right) \tilde{u}_{N}^{n}, v\right) .
\end{aligned}
$$

Subtracting equation (62) from equation (61), due to

$$
\begin{gathered}
\delta_{\hat{t}} u^{* n}-\delta_{\hat{t}} u_{N}^{n}=\frac{u^{* n+1}-u^{* n-1}}{2 \tau}-\frac{u_{N}^{n+1}-u_{N}^{n-1}}{2 \tau}=\frac{\eta^{n+1}-\eta^{n-1}}{2 \tau}, \\
-\delta_{t}^{2} u^{n}+\delta_{t}^{2} u_{N}^{n}=-\frac{u^{n+1}-2 u^{n}+u^{n-1}}{\tau^{2}}+\frac{u_{N}^{n+1}-2 u_{N}^{n}+u_{N}^{n-1}}{\tau^{2}} \\
=-\frac{e^{n+1}-2 e^{n}+e^{n-1}}{\tau^{2}}, \\
(-\Delta)^{\alpha / 2} \tilde{u}_{N}^{n}-(-\Delta)^{\alpha / 2} \tilde{u}^{n} \\
=(-\Delta)^{\alpha / 2} \frac{u_{N}^{n-1}+u_{N}^{n+1}}{2}-(-\Delta)^{\alpha / 2} \frac{u^{n-1}+u^{n+1}}{2} \\
=-(-\Delta)^{\alpha / 2} \frac{\left(u^{n-1}-u_{N}^{n-1}\right)+\left(u^{n+1}-u_{N}^{n+1}\right)}{2} \\
=-(-\Delta)^{\alpha / 2} \frac{e^{n-1}+e^{n+1}}{2},
\end{gathered}
$$

thus,

$$
\begin{aligned}
i \kappa\left(\delta_{\hat{t}} \eta^{n}, v\right)= & -\left(\delta_{t}^{2} e^{n}, v\right)-M^{\alpha}\left((-\Delta)^{\alpha / 2} \tilde{e}^{n}, v\right) \\
& -\frac{\beta}{2}\left(\left(\left|u^{n-1}\right|^{2}+\left|u^{n+1}\right|^{2}\right) \tilde{u}^{n}\right. \\
& \left.-\left(\left|u_{N}^{n-1}\right|^{2}+\left|u_{N}^{n+1}\right|^{2}\right) \tilde{u}_{N}^{n}, v\right)+\left(R^{n}, v\right) .
\end{aligned}
$$

According to the orthogonality of operator $P_{N}$, i.e., $\left(P_{N}\right.$ $u-u, v)=0, \forall v \in S_{N}$. Therefore,

$$
\begin{aligned}
\left(e^{j}, \eta^{k}\right) & =\left(\xi^{j}+\eta^{j}, \eta^{k}\right)=\left(\xi^{j}, \eta^{k}\right)+\left(\eta^{j}, \eta^{k}\right) \\
& =\left(\eta^{j}, \eta^{k}\right), j, k=0,1, \cdots, N_{t} .
\end{aligned}
$$

Let $v=\delta_{\hat{t}} \eta^{n}$ in (64), and taking the real part, due to

$$
\operatorname{Re}\left(i \kappa\left(\delta_{\grave{t}} \eta^{n}, \delta_{\hat{t}} \eta^{n}\right)\right)=0
$$

$\operatorname{Re}\left(\delta_{t}^{2} e^{n}, \delta_{\hat{t}} \eta^{n}\right)=\operatorname{Re}\left(\delta_{t}^{2} \eta^{n}, \delta_{\hat{t}} \eta^{n}\right)$

$$
\begin{aligned}
& =\frac{1}{2 \tau} \operatorname{Re}\left(\delta_{t} \eta^{n}-\delta_{t} \eta^{n-1}, \delta_{t} \eta^{n}+\delta_{t} \eta^{n-1}\right) \\
& =\frac{\left\|\delta_{t} \eta^{n}\right\|^{2}-\left\|\delta_{t} \eta^{n-1}\right\|^{2}}{2 \tau},
\end{aligned}
$$

$$
\begin{aligned}
\operatorname{Re} & \left((-\Delta)^{\alpha / 2} \tilde{e}^{n}, \delta_{\tilde{t}} \eta^{n}\right) \\
= & \operatorname{Re}\left((-\Delta)^{\alpha / 2} \tilde{\eta}^{n}, \delta_{\hat{t}} \eta^{n}\right) \\
= & \operatorname{Re}\left((-\Delta)^{\alpha / 2} \frac{\eta^{n-1}+\eta^{n+1}}{2}, \frac{\eta^{n+1}-\eta^{n-1}}{2 \tau}\right) \\
= & \frac{1}{4 \tau}\left(\left((-\Delta)^{\alpha / 4} \eta^{n+1},(-\Delta)^{\alpha / 4} \eta^{n+1}\right)\right. \\
& \left.-\left((-\Delta)^{\alpha / 4} \eta^{n-1},(-\Delta)^{\alpha / 4} \eta^{n-1}\right)\right) \\
= & \frac{\left\|(-\Delta)^{\alpha / 4} \eta^{n+1}\right\|^{2}-\left\|(-\Delta)^{\alpha / 4} \eta^{n-1}\right\|^{2}}{4 \tau},
\end{aligned}
$$

therefore, using (66)-(68), this implies

$$
\begin{aligned}
\operatorname{Re}\left(R^{n}, \delta_{\grave{t}} \eta^{n}\right)= & \frac{\left\|\delta_{t} \eta^{n}\right\|^{2}-\left\|\delta_{t} \eta^{n-1}\right\|^{2}}{2 \tau}+\frac{M^{\alpha}}{4 \tau}\left(\left\|(-\Delta)^{\alpha / 4} \eta^{n+1}\right\|^{2}\right. \\
& \left.-\left\|(-\Delta)^{\alpha / 4} \eta^{n-1}\right\|^{2}\right)+\operatorname{Re}\left(G^{n}, \delta_{\widehat{t}} \eta^{n}\right),
\end{aligned}
$$

where

$$
\begin{aligned}
\left|G^{n}\right|= & \frac{\beta}{2}\left(\left(\left|u^{n-1}\right|^{2}+\left|u^{n+1}\right|^{2}\right) \tilde{u}^{n}-\left(\left|u_{N}^{n-1}\right|^{2}+\left|u_{N}^{n+1}\right|^{2}\right) \tilde{u}_{N}^{n}\right) \\
= & \mid \frac{\beta}{2}\left(\left|u^{n-1}\right|^{2}+\left|u^{n+1}\right|^{2}\right) \frac{u^{n-1}+u^{n+1}}{2} \\
& -\frac{\beta}{2}\left(\left|u^{n-1}-e^{n-1}\right|^{2}+\left|u^{n+1}-e^{n+1}\right|^{2}\right) \frac{u_{N}^{n-1}+u_{N}^{n+1}}{2} \mid
\end{aligned}
$$




$$
\begin{aligned}
= & \mid \frac{\beta}{2}\left(\left|u^{n-1}\right|^{2} \tilde{e}^{n}+\left|u^{n+1}\right|^{2} \tilde{e}^{n}+e^{n-1} \bar{u}_{N}^{n-1} \tilde{u}_{N}^{n}\right. \\
& \left.+u^{n-1} \bar{e}^{n-1} \tilde{u}_{N}^{n}+e^{n+1} \bar{u}_{N}^{n+1} \tilde{u}_{N}^{n}+u^{n+1} \bar{e}^{n+1} \tilde{u}_{N}^{n}\right) \mid \\
\leq & \frac{\beta}{2}\left(\max \left\{\left|u^{n-1}\right|,\left|u^{n+1}\right|,\left|u_{N}^{n-1}\right|,\left|u_{N}^{n+1}\right|\right\}^{2}\right. \\
& \left.\cdot\left(2\left|e^{n-1}\right|+2\left|e^{n+1}\right|+2\left|\tilde{e}^{n}\right|\right)\right) .
\end{aligned}
$$

Thus, according to Lemma 6, we can get

$$
\left|G^{n}\right|^{2} \leq C\left(\left|e^{n-1}\right|^{2}+\left|e^{n+1}\right|^{2}\right)
$$

Note Lemma 1; it gives that

$$
\begin{aligned}
\left\|G^{n}\right\|^{2} & =\int_{\Omega}\left|G^{n}\right|^{2} d x \leq C\left(\left\|e^{n-1}\right\|^{2}+\left\|e^{n+1}\right\|^{2}\right) \\
& \leq C\left(\left\|\eta^{n-1}\right\|^{2}+\left\|\eta^{n+1}\right\|^{2}\right)+C N^{-2 s}\|u\|_{s}^{2}
\end{aligned}
$$

Then,

$$
\begin{aligned}
\operatorname{Re}\left(G^{n}, \delta_{\widehat{t}} \eta^{n}\right) \leq & \frac{\left\|G^{n}\right\|^{2}+\left\|\delta_{\widehat{t}} \eta^{n}\right\|^{2}}{2} \\
\leq & \frac{1}{2}\left\|\delta_{\widehat{t}} \eta^{n}\right\|^{2}+C\left(\left\|\eta^{n-1}\right\|^{2}+\left\|\eta^{n+1}\right\|^{2}\right) \\
& +C N^{-2 s}\|u\|_{s}^{2},
\end{aligned}
$$$$
\operatorname{Re}\left(R^{n}, \delta_{\widehat{t}} \eta^{n}\right) \leq \frac{\left\|R^{n}\right\|^{2}+\left\|\delta_{\widehat{t}} \eta^{n}\right\|^{2}}{2} \leq \frac{1}{2}\left\|\delta_{\hat{t}} \eta^{n}\right\|^{2}+C\left(\tau^{4}\right) \text {. }
$$

Thus, (69) becomes

$$
\begin{aligned}
& \frac{\left\|\delta_{t} \eta^{n}\right\|^{2}-\left\|\delta_{t} \eta^{n-1}\right\|^{2}}{2 \tau} \\
& \quad+\frac{M^{\alpha}}{4 \tau}\left(\left\|(-\Delta)^{\alpha / 4} \eta^{n+1}\right\|^{2}-\left\|(-\Delta)^{\alpha / 4} \eta^{n-1}\right\|^{2}\right) \\
& \leq C\left(\left\|\eta^{n-1}\right\|^{2}+\left\|\eta^{n+1}\right\|^{2}\right)+\left\|\delta_{\overparen{t}} \eta^{n}\right\|^{2}+C\left(\tau^{4}+N^{-2 s}\|u\|_{s}^{2}\right) .
\end{aligned}
$$

Because of

$$
\delta_{\widehat{t}} \eta^{n}=\frac{\delta_{t} \eta^{n}+\delta_{t} \eta^{n-1}}{2}
$$

and from Lemma 5, it gives that

$$
\frac{\left\|\eta^{n}\right\|^{2}-\left\|\eta^{n-1}\right\|^{2}}{\tau} \leq\left\|\delta_{t} \eta^{n}\right\|^{2}+\frac{1}{2}\left(\left\|\eta^{n}\right\|^{2}+\left\|\eta^{n-1}\right\|^{2}\right) .
$$

Then, combining (74) and (76) leads to

$$
\begin{aligned}
& \frac{\left\|\delta_{t} \eta^{n}\right\|^{2}-\left\|\delta_{t} \eta^{n-1}\right\|^{2}}{2 \tau}+\frac{M^{\alpha}}{4 \tau}\left(\left\|(-\Delta)^{\alpha / 4} \eta^{n+1}\right\|^{2}\right. \\
& \left.-\left\|(-\Delta)^{\alpha / 4} \eta^{n-1}\right\|^{2}\right)+\frac{\left\|\eta^{n}\right\|^{2}-\left\|\eta^{n-1}\right\|^{2}}{\tau} \\
& \leq C\left(\left\|\delta_{t} \eta^{n}\right\|^{2}+\left\|\delta_{t} \eta^{n-1}\right\|^{2}+\left\|\eta^{n}\right\|^{2}+\left\|\eta^{n+1}\right\|^{2}+\left\|\eta^{n-1}\right\|^{2}\right) \\
& \quad+C\left(\tau^{4}+N^{-2 s}\|u\|_{s}^{2}\right) .
\end{aligned}
$$

Summing above inequalities (77) from 1 to $n$ yields

$$
\begin{gathered}
\frac{1}{2}\left\|\delta_{t} \eta^{n}\right\|^{2}+\frac{M^{\alpha}}{4}\left(\left\|(-\Delta)^{\alpha / 4} \eta^{n+1}\right\|^{2}+\left\|(-\Delta)^{\alpha / 4} \eta^{n}\right\|^{2}\right)+\left\|\eta^{n}\right\|^{2} \\
\leq \tau C \sum_{i=1}^{n}\left(\frac{1}{2}\left\|\delta_{t} \eta^{i}\right\|^{2}+\frac{M^{\alpha}}{4}\left(\left\|(-\Delta)^{\alpha / 4} \eta^{i+1}\right\|^{2}\right.\right. \\
\left.\left.+\left\|(-\Delta)^{\alpha / 4} \eta^{i}\right\|^{2}\right)+\left\|\eta^{i}\right\|^{2}\right)+C\left(\tau^{4}+N^{-2 s}\|u\|_{s}^{2}\right)
\end{gathered}
$$

Hence, using the discrete Gronwall's inequality gives

$$
\begin{aligned}
& \frac{1}{2}\left\|\delta_{t} \eta^{n}\right\|^{2}+\frac{M^{\alpha}}{4}\left(\left\|(-\Delta)^{\alpha / 4} \eta^{n+1}\right\|^{2}+\left\|(-\Delta)^{\alpha / 4} \eta^{n}\right\|^{2}\right)+\left\|\eta^{n}\right\|^{2} \\
& \quad \leq C\left(\tau^{4}+N^{-2 s}\|u\|_{s}^{2}\right)
\end{aligned}
$$

thus,

$$
\left\|\eta^{n}\right\|^{2} \leq C\left(\tau^{4}+N^{-2 s}\|u\|_{s}^{2}\right)
$$

Substituting (80) into (60) can yield

$$
\left\|e^{n}\right\| \leq C\left(N^{-s}\|u\|_{s}+\tau^{2}\right)
$$

which immediately gives conclusion.

Similar to the proof of Theorem 7, we can obtain the following theorem.

Theorem 8. Let $s \geq 1$; assume that $u \in C^{2}\left(I ; H_{p e r}^{\alpha}(\Omega) \cap H^{s}\right.$ $(\Omega))$ is the exact solution of (6)-(8), and $u_{N}^{n}$ is the numerical solution of (32). It possesses the following conclusion:

$$
\left\|u_{N}^{n}-u\left(x, t_{n}\right)\right\| \leq C\left(\tau^{2}+N^{-s}\|u\|_{s}\right) .
$$

\section{Numerical Example}

Numerical examples will be proposed in this section to verify the correctness of the theoretical analysis, that is, the convergence of the numerical method and its ability to maintain discrete mass and discrete energy. 


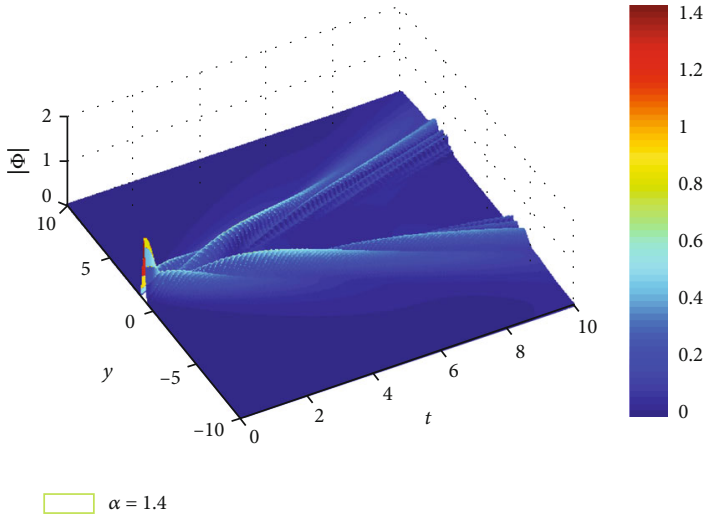

FIGURE 1: Numerical solutions for equation (83) with $\alpha=1.4$ when $\tau=0.01$ and $N=128$.

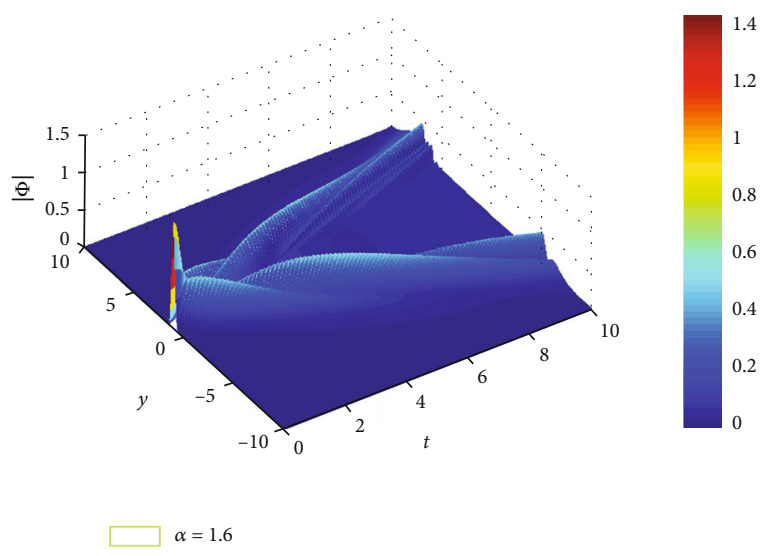

Figure 2: Numerical solutions for equation (83) with $\alpha=1.6$ when $\tau=0.01$ and $N=128$.

Example 1. Consider the nonlinear fractional Schrödinger equation with the wave operator:

$$
\left\{\begin{array}{l}
\phi_{t t}+(-\Delta)^{\alpha / 2} \phi+i \phi_{t}+|\phi(y, t)|^{2} \phi(y, t)=0, \quad y \in(-10,10), t \in(0, T], \\
\phi(-10, t)=\phi(10, t)=0, \quad t \in[0, T], \\
\phi(y, 0)=(1+i) y \exp \left(-10(1-y)^{2}\right), \phi_{t}(y, 0)=0, \quad y \in(-10,10) .
\end{array}\right.
$$

Let $\tau=0.01, N=128$, and $T=10$. Figures 1 and 2 present the numerical solutions for $\alpha=1.4$ and $\alpha=1.6$. We can find that the order of $\alpha$ will affect the shape of the solution.

There is no exact solution of (83) known for $1<\alpha<2$. Therefore, numerical solution calculated by the method (24) with $N=1024$ and $\tau=2^{-10}$ is taken as the reference solution. Let $\Phi$ be the numerical solution, and calculate the error at $t=t_{n}$ in the sense of the discrete $L^{2}$ norm:

$$
\text { error }=\left\|\phi^{n}-\Phi^{n}\right\|
$$

The convergence rates in the direction of time and space are calculated as
TABLE 1: Errors and convergence rates in time for $N=512$ and $T=1$.

\begin{tabular}{cccc}
\hline$\alpha$ & $\tau$ & Error & Order \\
\hline \multirow{3}{*}{1.4} & $2^{-5}$ & $1.9071 e-2$ & - \\
& $2^{-6}$ & $4.7789 e-3$ & 1.9966 \\
& $2^{-7}$ & $1.1822 e-3$ & 2.0152 \\
\hline \multirow{2}{*}{1.6} & $2^{-5}$ & $3.2228 e-2$ & - \\
& $2^{-6}$ & $7.8070 e-3$ & 1.9952 \\
& $2^{-7}$ & $1.9320 e-3$ & 2.0148 \\
\hline
\end{tabular}

TABLE 2: Errors and convergence rates in space for $\tau=2^{-10}$ and $T=1$.

\begin{tabular}{cccc}
\hline$\alpha$ & $N$ & Error & Order \\
\hline \multirow{4}{*}{1.4} & 128 & $1.3922 e-2$ & - \\
& 256 & $8.4405 e-3$ & 0.7220 \\
& 512 & $3.9789 e-3$ & 1.0850 \\
\hline \multirow{2}{*}{1.6} & 128 & $1.9930 e-2$ & - \\
& 256 & $1.2081 e-2$ & 0.7225 \\
& 512 & $5.6949 e-3$ & 1.0850 \\
\hline
\end{tabular}

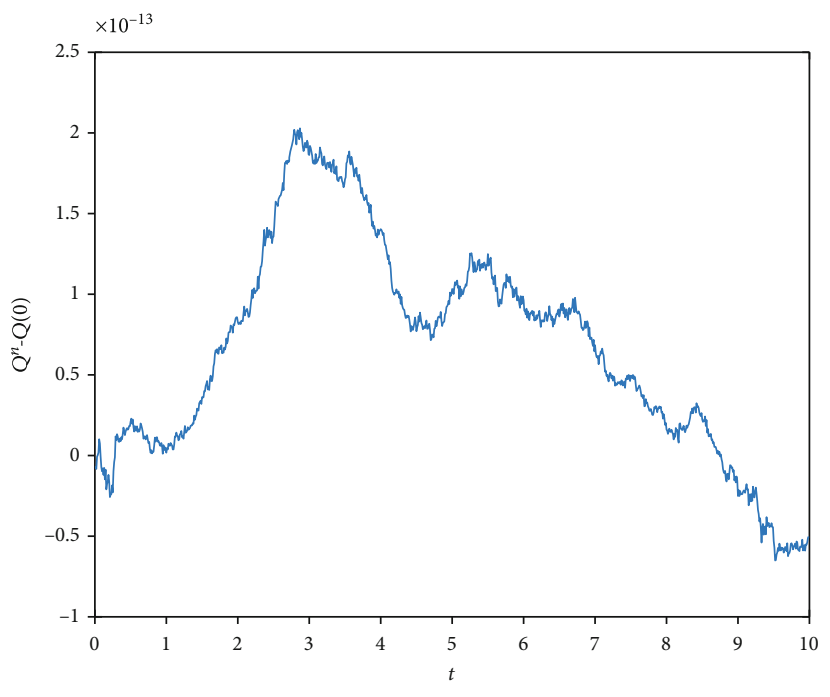

FIGURE 3: Discrete mass error when $\tau=0.01, N=128$, and $\alpha=1.4$.

$$
\text { order }=\left\{\begin{array}{l}
\frac{\log \left(\left\|\operatorname{error}\left(\tau_{1}\right)\right\| /\left\|\operatorname{error}\left(\tau_{2}\right)\right\|\right)}{\log \left(\tau_{1} / \tau_{2}\right)}, \\
\frac{\log \left(\left\|\operatorname{error}\left(N_{1}\right)\right\| /\left\|\operatorname{error}\left(N_{2}\right)\right\|\right)}{\log \left(N_{1} / N_{2}\right)} .
\end{array}\right.
$$

Let $T=1$. Tables 1 and 2 show that the numerical method is proven to have spectral accuracy in space and second-order accuracy in time for solving equation (83) with $\alpha=1.4$ and $\alpha=1.6$. 


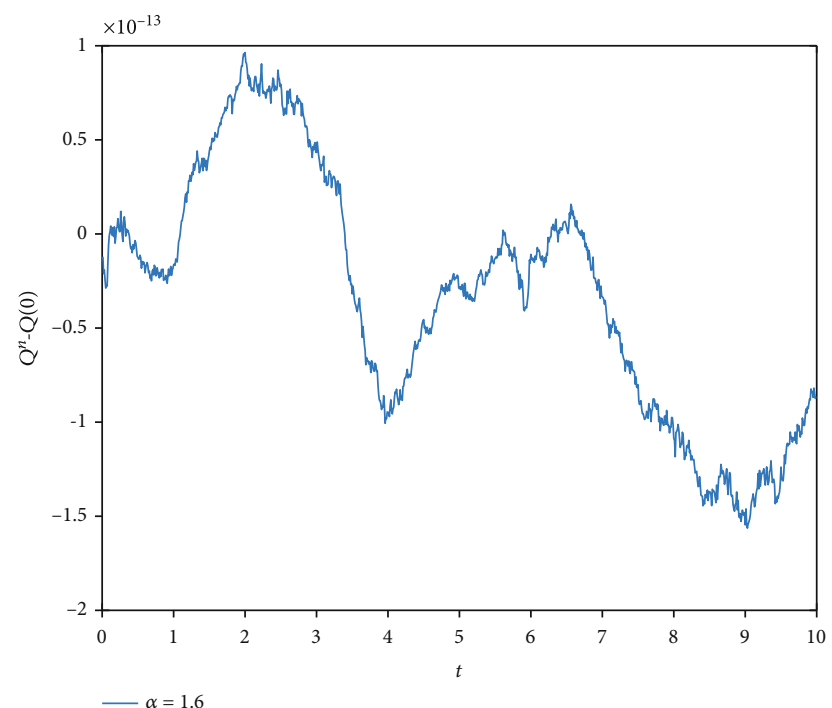

Figure 4: Discrete mass error when $\tau=0.01, N=128$, and $\alpha=1.6$.

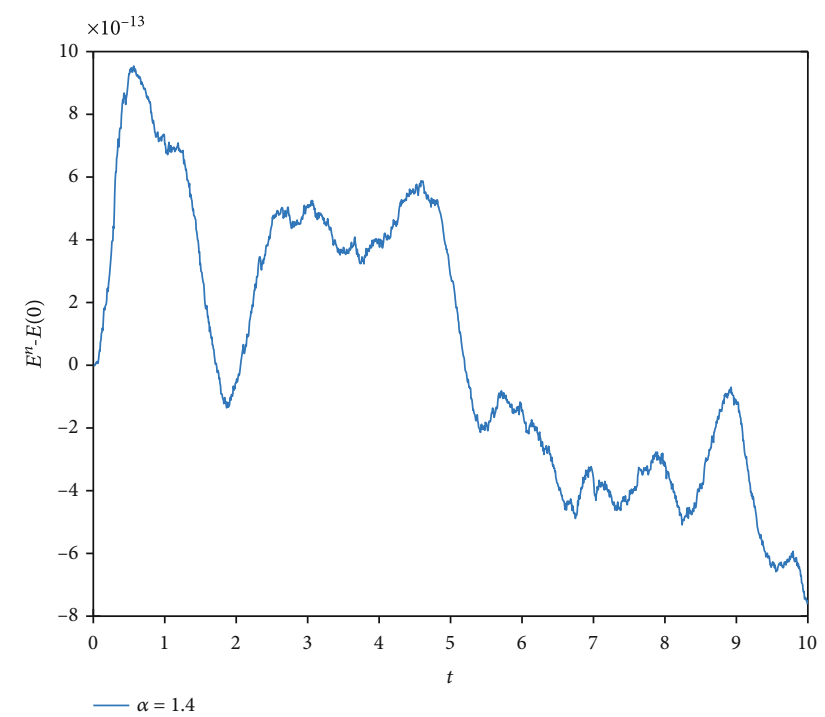

Figure 5: Discrete energy error when $\tau=0.01, N=128$, and $\alpha=1.4$.

Figures 3-6 show the ability of the numerical method (24) to maintain the discrete mass and discrete energy when $\alpha=1.4$ and $\alpha=1.6$. It can be seen from the figure that the numerical method (24) maintains the discrete mass and discrete energy well.

\section{Conclusion}

For the fractional Schrödinger equation with wave operators, we successfully constructed the effective conservative CrankNicolson Fourier spectral method for solving this equation, based on the relative theory of a fractional-order derivative and its property. We give the strict theoretical derivation for the convergence rate of the numerical method, i.e., $O\left(\tau^{2}\right.$ $+N^{-s}\|u\|_{s}$ ). Finally, numerical examples are introduced to verify the correctness of the theoretical results and the validity of our numerical methods. Both theoretical derivation

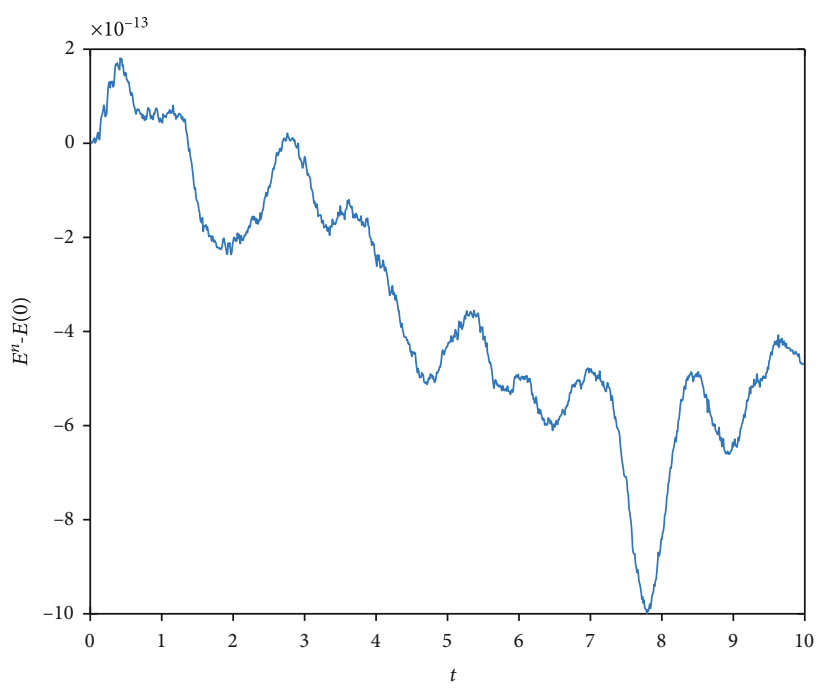

$-\alpha=1.6$

FIgURE 6: Discrete energy error when $\tau=0.01, N=128$, and $\alpha=1.6$.

and numerical experiment verify that the numerical method can keep the energy conservation and mass conservation of the original fractional Schrödinger equation. Both environmental noise and regime switching are important factors [21-29]; we will introduce them in the model $((6)-(8))$ in the future.

\section{Data Availability}

No data were used to support this study.

\section{Conflicts of Interest}

The authors declare that they have no conflicts of interest.

\section{Acknowledgments}

This work is supported by the National Natural Science Foundation of China under grant no. 11901329, the Natural Science Foundation of Shandong Province under grant nos. ZR2019BA022 and ZR2020QA008, and the Teaching Reform Projects of Qingdao University of Technology (F2019-044 and F2020-079).

\section{References}

[1] Y. Gong, Q. Wang, Y. Wang, and J. Cai, “A conservative Fourier pseudo-spectral method for the nonlinear Schrodinger equation," Journal of Computational Physics, vol. 328, pp. 354-370, 2017.

[2] L. Kong, J. Zhang, Y. Cao, Y. Duan, and H. Huang, "Semiexplicit symplectic partitioned Runge-Kutta Fourier pseudospectral scheme for Klein-Gordon-Schrodinger equations," Computer Physics Communications, vol. 181, no. 8, pp. 13691377, 2010.

[3] H. Li, Z. Mu, and Y. Wang, "An energy-preserving CrankNicolson Galerkin spectral element method for the two dimensional nonlinear Schrodinger equation," Journal of 
Computational and Applied Mathematics, vol. 344, pp. 245258, 2018.

[4] X. Zhang, J. Jiang, Y. Wu, and Y. Cui, "The existence and nonexistence of entire large solutions for a quasilinear Schrodinger elliptic system by dual approach," Applied Mathematics Letters, vol. 100, p. 106018, 2020.

[5] H. Li, C. Jiang, and Z. Lv, "A Galerkin energy-preserving method for two dimensional nonlinear Schrodinger equation," Applied Mathematics and Computation, vol. 324, pp. 16-27, 2018.

[6] X. Zhang, J. Jiang, Y. Wu, and B. Wiwatanapataphee, "Iterative properties of solution for a general singular $\mathrm{n}$-Hessian equation with decreasing nonlinearity," Applied Mathematics Letters, vol. 112, p. 106826, 2021.

[7] X. Zhang, J. Xu, J. Jiang, Y. Wu, and Y. Cui, “The convergence analysis and uniqueness of blow-up solutions for a Dirichlet problem of the general k-Hessian equations," Applied Mathematics Letters, vol. 102, p. 106124, 2020.

[8] M. Li, C. Huang, and P. Wang, "Galerkin finite element method for nonlinear fractional Schrödinger equations," Numerical Algorithms, vol. 74, no. 2, pp. 499-525, 2017.

[9] M. Li, C. Huang, and Z. Zhang, "Unconditional error analysis of Galerkin FEMs for nonlinear fractional Schrödinger equation," Applicable Analysis, vol. 97, no. 2, pp. 295-315, 2018.

[10] Q. Liu, F. Zeng, and C. Li, "Finite difference method for timespace-fractional Schrödinger equation," International Journal of Computer Mathematics, vol. 92, no. 7, pp. 1439-1451, 2015.

[11] Y. Shi, Q. Ma, and X. Ding, "A new energy-preserving scheme for the fractional Klein-Gordon-Schrödinger equations," Advances in Applied Mathematics and Mechanics, vol. 11, no. 5, pp. 1219-1247, 2019.

[12] T. J. Bridges and S. Reich, "Multi-symplectic spectral discretizations for the Zakharov-Kuznetsov and shallow water equations," Physica D: Nonlinear Phenomena, vol. 152-153, pp. 491-504, 2001.

[13] J. Chen and M. Qin, "Multi-symplectic Fourier pseudospectral method for the nonlinear Schrödinger equation," Electronic Transactions on Numerical Analysis, vol. 12, pp. 193204, 2001.

[14] J. Cai and Y. Wang, "A conservative Fourier pseudospectral algorithm for a coupled nonlinear Schrödinger system," Chinese Physics B, vol. 22, no. 6, article 060207, 2013.

[15] Y. Gong, J. Cai, and Y. Wang, "Multi-symplectic Fourier pseudospectral method for the Kawahara equation," Communications in Computational Physics, vol. 16, no. 1, pp. 35-55, 2014.

[16] R. Yan, M. Han, Q. Ma, and X. Ding, “A spectral collocation method for nonlinear fractional initial value problems with a variable-order fractional derivative," Computation \& Applied Mathematics, vol. 38, no. 2, 2019.

[17] M. Ainsworth and Z. Mao, "Analysis and approximation of a fractional Cahn-Hilliard equation," SIAM Journal on Numerical Analysis, vol. 55, no. 4, pp. 1689-1718, 2017.

[18] X. Ye, "The Fourier collocation method for the Cahn-Hilliard equation," Computers and Mathematics with Applications, vol. 44, no. 1-2, pp. 213-229, 2002.

[19] J. Shen, T. Tang, and L. Wang, Spectral Methods: Algorithms, Analysis and Applications, Springer, London, 2011.

[20] L. Zhang and Q. Chang, "A conservative numerical scheme for a class of nonlinear Schrodinger equation with wave operator," Applied Mathematics and Computation, vol. 145, no. 2-3, pp. 603-612, 2003.
[21] M. Liu and C. Bai, "Optimal harvesting of a stochastic mutualism model with regime-switching," Applied Mathematics and Computation, vol. 373, p. 125040, 2020.

[22] C. Lu, "Dynamics of a stochastic Markovian switching predator-prey model with infinite memory and general Levy jumps," Mathematics and Computers in Simulation, vol. 181, pp. 316-332, 2021.

[23] B. Han, D. Jiang, T. Hayat, A. Alsaedi, and B. Ahmad, "Stationary distribution and extinction of a stochastic staged progression AIDS model with staged treatment and second-order perturbation," Chaos, Solitons \& Fractals, vol. 140, article 110238, 2020.

[24] C. Lu and X. Ding, "Periodic solutions and stationary distribution for a stochastic predator-prey system with impulsive perturbations," Applied Mathematics and Computation, vol. 350, pp. 313-322, 2019.

[25] C. Lu, G. Sun, and Y. Zhang, "Stationary distribution and extinction of a multi-stage HIV model with nonlinear stochastic perturbation," Journal of Applied Mathematics and Computing, 2021.

[26] M. Song, W. Zuo, D. Jiang, and T. Hayat, "Stationary distribution and ergodicity of a stochastic cholera model with multiple pathways of transmission," Journal of the Franklin Institute, vol. 357, no. 15, pp. 10773-10798, 2020.

[27] C. Lu and X. Ding, "Dynamical behavior of stochastic delay Lotka-Volterra competitive model with general Lévy jumps," Physica A: Statistical Mechanics and its Applications, vol. 531, article 121730, 2019.

[28] G. Lan, S. Yuan, and B. Song, "The impact of hospital resources and environmental perturbations to the dynamics of SIRS model," Journal of the Franklin Institute, vol. 358, no. 4, pp. 2405-2433, 2021.

[29] Z. Wang, M. Deng, and M. Liu, "Stationary distribution of a stochastic ratio-dependent predator-prey system with regime-switching," Chaos, Solitons \& Fractals, vol. 142, article 110462, 2021. 